\title{
Micromundos, Construccionismo y Matemáticas ${ }^{1}$
}

\author{
Microworlds, Constructionism and Maths ${ }^{2}$
}

\section{Richard Noss ${ }^{3}$ \\ Celia Hoyles ${ }^{4}$}

\begin{abstract}
Resumen: En este artículo, esbozamos la idea del construccionismo y cómo puede ser puesta en práctica a través del diseño de "micromundos", islas aisladas y accesibles de actividad, dónde se encuentren pepitas de conocimiento relevante a través de herramientas y secuencias de actividades especialmente diseñadas y con pedagogías adecuadamente orientadas. En la segunda parte del artículo, describimos el diseño, implementación y evaluación de una intervención construccionista, ScratchMaths, introducida en Inglaterra, país en el que la computación es obligatoria para todos los niveles educativos (de los 5 a los 16 años). Este estudio de caso pone de manifiesto la tensión entre la fidelidad de una innovación al implementarla y su adaptación por parte de los
\end{abstract}

Fecha de recepción: 16 de enero de 2019. Fecha de aceptación: 2 de abril de 2019.

1 Este artículo se basa en parte en el de "Constructionism and Microworlds", publicado en Duval, E., Sharples, M., \& Sutherland, R. (2017). (Eds.). Technology Enhanced Learning: Research Themes (pp. 29-35). Cham, Switzerland: Springer. doi: 10.1007/978-3-319-02600-8_3

2 Traducción del inglés: Ana Isabel Sacristán Rock

3 UCL Knowledge Lab, UCL Institute of Education, University College London, r.noss@ucl.ac.uk, orcid. org/0000-0001-6301-6489

${ }^{4}$ UCL Knowledge Lab, UCL Institute of Education, University College London, c.hoyles@ucl.ac.uk, orcid. org/0000-0001-5724-7600 
profesores, especialmente en el contexto de las matemáticas, la cuál es una materia muy exigente tanto para los docentes como para los alumnos.

Palabras clave: construccionismo; micromundos; ScratchMaths; fidelidad

\begin{abstract}
In this paper, we outline the notion of constructionism and how it might be put into practice through the design of "microworlds", insulated and accessible islands of activity in which nuggets of relevant knowledge are encountered through specially designed tools, sequences of activities with suitably oriented pedagogies. In the second part of the paper, we describe the design, implementation and evaluation of a constructionist intervention, ScratchMaths, introduced in England where computing is compulsory throughout schooling (from 5 to 16 years). This case study highlights the tension between the fidelity of implementation of an innovation and its adaptation by teachers, especially the context of mathematics, which is high stakes for both teachers and learners.
\end{abstract}

Keywords: constructionism; microworlds; ScratchMaths; fidelity

\title{
1. INTRODUCCIÓN
}

Empezamos con una observación que Seymour Papert escribió en la cubierta del libro Geometría de la Tortuga (Abelson and diSessa, 1981) donde explica que ese libro representa "el primero de una nueva generación de libros de texto de matemáticas". Parece que eso era una expectativa demasiado grande: aún el observador más optimista tendría que admitir que los cambios no se dieron tal como Papert lo contempló. Pero cabe notar que en su observación de que habría algo sobre lo cual se querrían escribir libros y que la gente los leyera, hay un currículo (o al menos un currículo parcial) implícito.

¿Qué tan exitosos han sido esta "nueva generación" de potenciales currículos y libros de texto para lograr las metas de un cambio fundamental de lo que se puede aprender matemáticamente? Un juicio es claramente una cuestión de perspectiva y, para nosotros, el cambio se puede considerar como fundamental hasta el grado en que se ha basado en dos ideas clave: la idea del construccionismo, y la idea de micromundo. Y como todas las grandes ideas, ambas han sido sujetas 
a presiones para diluir lo fundamental a favor de lo pragmático. Entender lo que esto implica y qué tan lejos hemos llegado en estos últimos cuarenta años nos ayudará a entender por qué el cambio fundamental es tan difícil.

\section{2. ¿QUÉ ES EL CONSTRUCCIONISMO?}

Fue hace aproximadamente 50 años que Seymour Papert lanzó la idea del construccionismo. La idea central es que es una manera poderosa para que los estudiantes construyan estructuras de conocimiento en su mente, que construyan representaciones externas, entidades físicas o virtuales sobre las cuales se pueda reflexionar y que puedan ser editadas y compartidas. La interpretación más amplia, relatada continuamente en revistas científicas y populares, es que el construccionismo es lo mismo que el constructivismo: no lo es.

El construccionismo [...] tiene la misma connotación del constructivismo del aprendizaje como "creación de estructuras de conocimiento", independientemente de las circunstancias del aprendizaje. Luego agrega la idea de que esto ocurre en forma especialmente oportuna en un contexto donde la persona que aprende está conscientemente dedicada a construir una entidad pública, ya sea un castillo de arena en la playa o una teoría del universo. (Papert, 1991/2002, p. 2)

A diferencia del constructivismo, el construccionismo busca, por lo tanto, formar una teoría de la pedagogía, al abordar directamente la pregunta de cómo ayudar mejor a los estudiantes a aprender. En contraste, el constructivismo es una teoría de cómo la gente aprende, independientemente de las circunstancias de ese aprendizaje, o de si se llega, o no, a involucrar la enseñanza (para una introducción al constructivismo véase, por ejemplo, von Glasersfeld, 1989). Como lo plantea Papert, "la palabra con n", el construccionismo, más que "la palabra con $\checkmark$ ', el constructivismo, aspira a intentar teorizar estrategias que alineen las maneras en que la gente aprende, con las maneras en que hace sentido ayudarles a entender, especialmente a través del diseño de artefactos adecuados. La palabra "especialmente" es aquí crucial, puesto que centra la atención en el diseño: en el diseño de ambientes construccionistas, lo cual lleva a la noción de micromundo que discutiremos más adelante.

Un ejemplo clásico de ambiente construccionista es uno que se centra en Logo, el lenguaje de programación derivado del lenguaje de inteligencia artificial, 
LISP (Harvey, 1997). Logo era, y sigue siendo en sus diversas encarnaciones, un lenguaje de programación completo, a través del cual gente de todas las edades puede programar cualquier cosa que pueda imaginar -una imagen, un robot, un video juego o una pieza musical. En su más reciente encarnación en Scratch, cuenta con millones de adultos y niños publicando y "reinventando" lo que es esencialmente código Logo/Lisp con una intuitiva y manipulativa interfaz visual basada en bloques.

La tortuga (la cual fue inventada algunos años después del lanzamiento del primer Logo) tiene muchas facetas, primordialmente como un medio a través del cual pueden participar incluso niños pequeños con ideas que son fundamentales para las matemáticas (tales como la geometría diferencial) y que de otra manera estarían fuera de su alcance. Por ejemplo, algunas de las ideas fundamentales de la geometría diferencial, como curvatura, se alega que pueden ser más accesibles a través de un medio expresivo que saque provecho de la sintonicidad corporal. La presencia de objetos manipulables "concretos" abre tres potencialidades distintas pero relacionadas, para el alumno.

Primero, el ambiente construccionista busca presentar un medio atractivo donde explorar y aprender de la retroalimentación (en diferentes formas), de la misma manera en que uno puede dominar un idioma extranjero al vivir en el país adecuado. Segundo, el alumno puede adoptar un acercamiento al aprendizaje basado en la construcción, diseñado de tal manera que los estudiantes se topen con "ideas poderosas" o pepitas intelectuales. La noción de ideas poderosas captura la noción de involucramiento con herramientas intelectuales, maneras de pensar que ofrecen al alumno acceso a conceptos y estrategias clave, que conectan con su propio conocimiento intuitivo. (Para una revisión comprensiva del papel de las herramientas en el aprendizaje de las matemáticas, véase Monaghan, Trouche \& Borwein, 2016).

Tercero, las herramientas construccionistas buscan ser expresivas, es decir que pueden ser adaptadas por sus usuarios para construir nuevas entidades, de maneras que surgen en la actividad. Al mismo tiempo, las herramientas restringen y dan forma a lo que los estudiantes pueden hacer, pensar y aprender y esas restricciones pueden diseñarse a modo de ser productivas para el aprendizaje. (Véase también el debate en torno a las nociones de abstracción situada, genesis instrumental y orquestación en Hoyles, Noss \& Kent, 2004).

Las potencialidades de Logo, nos permiten generalizar la idea del construccionismo más allá del caso de Logo y de sus descendientes. A medida que Logo evolucionó, el espacio digital de su entorno también evolucionó en 
tándem (iLogo fue inventado unos 30 años antes que la web!). Adicionalmente, la teoría del construccionismo ha adquirido más forma y detalle, inspirando a diseñadores a construir otras tecnologías que apoyan sus objetivos fundamentales: Boxer, Scratch, ${ }^{5}$ NetLogo, ${ }^{6}$ ToonTalk, ${ }^{7}$ y más recientemente hardware que finalmente ha llegado a ser omnipresente como el Raspberry Pi y el BBC Micro:bit. Numerosos ambientes enfocados al conocimiento han incursionado en la arena del construccionismo, con visiones similares del aprendizaje, tales como, en matemáticas, los sistemas de geometría dinámica (Sinclair \& Crespo, 2006) o, en música, Impromptu (Bamberger \& Hernández, 1999). Eisenberg (2003) también contribuyó a este conjunto, a través de sus descripciones de ambientes que mezclan materiales tradicionales y computacionales. A lo largo de los años, el construccionismo también ha aportado el marco de una línea fértil de investigación que detalla trayectorias de aprendizaje mediante herramientas, en una amplia gama de tópicos que van desde la topología hasta la composición musical.

La discusión previa ilustra que, tal como Papert se esforzó por resaltar, el construccionismo busca desarrollar estructuras de conocimiento en la mente junto con estructuras físicas o virtuales que son externas a la mente, y por ende es tanto una teoría epistemológica como una pedagogía (ver Harel \& Papert, 1991). Papert explica que la distinción entre el instruccionismo y el construccionismo también concierne a la epistemología y no es sólo acerca de dos maneras de pensar sobre la transmisión del conocimiento. Más bien, la distinción va más allá de la adquisición de conocimiento, para abordar la naturaleza del conocimiento y la naturaleza del conocer (Papert, 1993/1995). En otras palabras, el construccionismo implica escoger o designar representaciones, involucrando artefactos y pedagogías adecuadamente orientadas que conjuntamente puedan brindar un cambio fundamental al cómo aprender y al qué se aprende.

Una discusión estimulante de la reflexión sobre este cambio epistemológico fue explorada por Wilensky y Papert, quienes alegan que el construccionismo ha:

cambiado el enfoque de los medios al objeto de aprendizaje... cómo la estructura y las propiedades del conocimiento afectan las posibilidades de aprendizaje y el poder

\footnotetext{
5 http://scratch.mit.edu/

${ }^{6}$ http://ccl.northwestern.edu/netlogo/

7 http://www.toontalk.com/
} 
que les otorgan a individuos y a grupos. (Wilensky \& Papert, 2010, p. 1; traducción del inglés $)^{8}$

El nombre que le dan a este proceso es reestructuración,

... la codificación del conocimiento en un dominio como una función de la infraestructura representacional que se usa para expresar ese conocimiento. Un cambio de una estructuración de un dominio a otro como consecuencia de ese cambio de infraestructura representacional, lo llamamos reestructuración. (ibíd., pp. 2-3, traducción del inglés)

El ejemplo que dan (Papert, 2006) es el cambio (aunque claramente no llevado a cabo con propósitos educativos) de los números romanos a arábigos, un cambio que hizo posible que casi todo mundo pudiera calcular de maneras hasta entonces recónditas. Nuestro reto es pensar más allá de este ejemplo, y buscar identificar en dónde la presencia de la computadora ha cambiado, no sólo cómo el conocimiento se extiende y desarrolla, sino la naturaleza del conocimiento mismo, en disciplinas científicas, socio-científicas y de humanidades (véase, por ejemplo, Resnick, 1995).

Uno de los retos persistentes para lograr la visión del construccionismo, es la tensión entre buscar enseñar un contenido especifico como, por ejemplo, de matemáticas o de música, y al mismo tiempo otorgar al alumno la experiencia de construir, crear, hacer, y resolver problemas explotando ese conocimiento de contenido. Esas dos metas no son, por supuesto, antitéticas, pero tampoco es obvio cómo se pueden alinear para propósitos pedagógicos. Una solución que ha evolucionado es el diseño de "micromundos", de "islas" aisladas y accesibles de actividad, donde se encuentren pepitas de conocimiento relevante de una manera "natural" a través del uso de herramientas digitales.

${ }^{8}$ Este puede ser un momento apropiado para dirigir al lector interesado al NetLogo de Wilensky, cuyas copias contienen no solo el lenguaje de programación mismo, sino también docenas o cientos de ideas matemáticas que son 'reestructuraciones' posibilitadas por la nueva herramienta. 


\section{MICROMUNDOS}

Hoyles (1993) describe la evolución de la idea de micromundo desde su génesis en la comunidad de inteligencia artificial, donde se usó para describir un dominio relativamente simple y restringido, en el cual los sistemas computacionales podrían resolver problemas, hasta un ambiente más ampliamente concebido que sirviera de representación concreta de un dominio o estructura de conocimiento. La estructura incluye herramientas que son extensibles (de tal manera que herramientas y objetos se puedan combinar para formar nuevas herramientas, pero que sean transparentes para que su forma de operar sea visible, y que sean ricas en diversas representaciones). Edwards (1998) contrasta esta visión "estructural" de un micromundo con una visión "funcional" que, a medida que los estudiantes exploran, construyen y aprenden de la retroalimentación, prioriza sus características al hacerse éstas evidentes en su uso.

Esta visión funcional enfatiza la importancia de la forma en que el conocimiento realmente crece en interacción con los estudiantes. Como señala diSessa (2006), la instrucción tradicional no logra incluir cómo se construye el conocimiento, pieza por pieza, y capa por capa. Hay aquí una dualidad: un micromundo exitoso es a la vez un universo epistemológico y uno emocional, un lugar donde se pueden explorar ideas poderosas (matemáticas, científicas o artísticas); pero exploradas "en seguridad", constituyendo una incubadora tanto en el sentido de fomentar el desarrollo conceptual, como siendo un lugar seguro para cometer errores y mostrar ignorancia. Y, centralmente en estos días, es un lugar donde las ideas se pueden compartir, reinventar y mejorar. (Para una discusión anterior sobre estos aspectos gemelos de involucrarse a través de construir y compartir, véase la idea de webbing discutida por Noss \& Hoyles, 2006). El componente emocional es más que incidental a la idea de micromundo: construir y compartir implica que los estudiantes aprecien lo que están construyendo y también aborden el aprendizaje como algo colaborativo. En cuanto al primer punto, el famoso ejemplo de Papert en el prefacio de su libro Desafío a la Mente (Papert, 1980/1981), cuenta una historia que no trata sólo de lo mucho que aprendió sobre matemáticas jugando con engranajes, sino de cómo "se enamoró" de los engranajes, un conocimiento íntimo y consumidor que utilizó como modelo para el aprendizaje futuro de las matemáticas. Hay, por supuesto, contextos distintos a las matemáticas y la ciencia que han sido sujetos de un análisis construccionista: véase la discusión relacionada al dibujo y la pintura, en Clayson (2008) y Gargarian (1993). 
Pero además de ser un desafío intelectual para una participación auténtica, hay problemas que son fundamentales para los objetivos generales de aprendizaje. En relación a las matemáticas, Confrey y sus colegas lo expresaron así:

La importancia de aprovechar la cultura juvenil no debe subestimarse para motivar y sostener el progreso educativo de los estudiantes. Esto es especialmente cierto para materias como la ciencia y las matemáticas, que cargan con un capital social considerable, pero que es fácil para los estudiantes descartar como irrelevantes, aburridas y difíciles en un mundo de imágenes digitales, animaciones, recuperación fácil de información y comunicación. Necesitamos entornos interesantes, en los que los estudiantes realmente necesiten matemáticas para alcanzar metas que les parezcan atractivas, y que sean matemáticas visibles para los estudiantes y expresadas en un idioma con el que puedan conectarse. (Confrey et al., 2010, p. 20; traducción del inglés)

La participación auténtica de los estudiantes sigue siendo un gran desafío para la educación matemática actual. No es sencillo diseñar actividades que alienten a los estudiantes a abordar los conceptos matemáticos deseados, de maneras que hagan evidente para el estudiante que éstos tienen utilidad. Es decir, aunque el involucrarse en micromundos tiene como intención orientar a los alumnos hacia maneras matemáticas de pensar a través de estructuras establecidas por el diseñador, los alumnos deben mantener cierta autonomía. Deben asumir la responsabilidad de sus acciones y de los resultados que producen. Esto significa, por supuesto, que el aprendizaje nunca ocurrirá exactamente como se planeó, lo que implica un reto inevitable: cómo equilibrar la actividad auto-motivada mientras que se maximiza la oportunidad de toparse con las ideas poderosas planeadas (véase la "paradoja del juego" en Noss y Hoyles, 1996).

El terreno del aprendizaje matemático está repleto de reestructuraciones del conocimiento matemático, un ejemplo es el conjunto de "breves" en cada número de la revista International Journal of Computers in Mathematical Learning o en la biblioteca de modelos que acompaña a NetLogo. Es cierto que no hay un "nuevo currículum"; de hecho, la sola idea de que debería haber una "versión" de las matemáticas diseñada para poder ser aprendida podría ser consternante para muchos matemáticos. Pero buenos ejemplos sí existen. Uno de ellos es el estudio de Sacristán \& Noss, 2008, que muestra cómo la formalización de la idea del infinito en forma de fragmentos de programas puede ser una forma efectiva de apreciar estas ideas profundas y a menudo inaccesibles. 
Consideraremos con mayor profundidad a continuación, un intento coordinado de construir y evaluar una intervención basada en la programación computacional, el programa ScratchMaths (SM), que comprende un conjunto de micromundos diseñados para la exploración de temas matemáticos clave para niños de 9 a 11 años.

\section{EL PROYECTO SCRATCHMATHS: UNA SECUENCIA DE MICROMUNDOS MATEMÁTICOS}

Primero planteamos el contexto del proyecto ScratchMaths, lo cual es importante para enmarcar su aplicabilidad y rango. Desde septiembre de 2014, todas las escuelas para estudiantes de 5 a 16 años de edad en Inglaterra deben impartir el currículo nacional obligatorio de computación, lo cual incluye diseñar y crear programas. Hay desafíos para implementar, con orientación limitada, cómo enseñar el contenido propuesto, los niveles específicos de conocimiento o de comprensión que los alumnos deben alcanzar en cada etapa del currículo y problemas conceptuales con los que posiblemente los alumnos se topen y cómo deben abordarse. Otros desafíos tratan de cómo encajar el nuevo contenido curricular en un calendario ya bastante lleno y, lo más crucial desde nuestra perspectiva, cómo forjar vínculos interdisciplinarios de la computación con otras áreas curriculares.

El proyecto ScratchMaths (SM) buscaba abordar algunos de estos retos a través de una intervención longitudinal de dos años en varias escuelas de Inglaterra, para promover el pensamiento computacional alineado con el pensamiento y el razonamiento matemáticos. Así, SM fue diseñado como un currículo que aborda aspectos clave de los currículos de computación y de matemáticas de primaria para ese grupo de edad. La intervención consta de seis micromundos, llamados "módulos", tres al año, y fue diseñada por investigadores que trabajaban de cerca con cuatro escuelas "diseño" para probar y refinar los recursos para los alumnos y maestros. (Véanse los recursos para alumnos y maestros libremente disponibles en https:/www.ucl.ac.uk/ioe/research/projects/scratchmaths).

Anticipamos que el papel del maestro fuera crítico para una implementación exitosa de la innovación y, por ello, en sesiones que designamos como obligatorias de desarrollo profesional, se dedicó un tiempo considerable para compartir el enfoque pedagógico general de SM, a través de lo que llamamos las "5Es": Explorar, Explicar, intercambiar (Exchange), concebir (Envisage) y relacionar 
(bridgE). El objetivo era que esas E's estructuraran conjuntamente el enfoque global de la clase con las diferentes actividades del currículo SM (ver Benton et al., 2017).

En el primer año del currículo para estudiantes de 9-10 años, los conceptos computacionales se presentaron en un primer plano a las ideas matemáticas que estaban implícitas en micromundos titulados Tiling Patterns (patrones de mosaicos), Beetle Geometry (geometría del Escarabajo) y Collaborating Sprites (Sprites en colaboración). En el Segundo año, se introdujo a los mismos estudiantes (ahora de 10-11 años) a conceptos matemáticos y razonamiento matemático de manera explícita a través de un enfoque de programación que incluía un conjunto de nuevos conceptos computacionales ${ }^{9}$ en los micromundos titulados: Building with Numbers (construir con números), Exploring Mathematical Relationships (exploración de relaciones matemáticas), y Coordinates and Geometry (coordenadas y geometría).

Dado el reto de implementar un nuevo currículo, se proporcionó a los maestros SM una orientación detallada para la navegación de los materiales, los cuales fueron cuidadosamente estructurados y eran progresivos. Sin embargo, el equipo SM reconoció una emergente tensión en su búsqueda por otorgar un apoyo amplio y la necesidad de apropiación y autonomía de los maestros, por lo cual se dio espacio a los docentes para personalizar los materiales de acuerdo a sus propias metas y las necesidades de sus estudiantes. Esta es una variación de la paradoja del juego a la cual nos referimos arriba; ahora frecuentemente se habla de ésta como la tensión, o brecha, entre la fidelidad y la adaptación. El desafío es, cuando menos, reducir esta brecha y, de manera crucial, evitar el surgimiento de "mutaciones letales" (Brown \& Campione, 1996), donde los objetivos de la intervención se pierdan en su implementación.

El proyecto SM fue evaluado por un equipo de investigadores independientes, quienes se centraron en datos cuantitativos recolectados a través de cuestionarios y resultados de pruebas, una de las cuales fue la prueba de pensamiento computacional (CT, por sus siglas en inglés) aplicada al final del primer año de prueba, y una segunda fue la prueba nacional de matemáticas Key Stage 2 para estudiantes de 11 años.

El más significativo de los primeros resultados fue que los niños en las escuelas ScratchMaths lograron mayor progreso en los puntajes de CT, en comparación con los niños en las escuelas de control. En segundo lugar, el puntaje

\footnotetext{
9 Por ejemplo, eventos, animaciones, estructuras de control, variables, operadores y expresiones.
} 
de CT fue mayor para los estudiantes con desventajas educativas, medido por un estándar que se utiliza en Inglaterra para ello, a través de determinar la elegibilidad para comidas escolares gratuitas de acuerdo al ingreso familiar. En resumen, los estudiantes desfavorecidos se beneficiaron más de SM, mostrando un rendimiento mayor en $\mathrm{CT}$ que el grupo de control. Y tercero, no hubo diferencias en los puntajes entre niños y niñas, un hallazgo que no es insignificante cuando se considera el contexto de la preocupación generalizada de que las niñas de todas las edades prefieren no participar en la programación.

Los evaluadores encontraron, sin embargo, que SM no tuvo un impacto en el rendimiento matemático, usando las pruebas Key Stage 2 para niños de 11 años al final del sexto grado. Este es un resultado decepcionante, aunque la medición de éxito a través de estas pruebas siempre sería problemática. Una evaluación del impacto en el aprendizaje de las matemáticas a través, por ejemplo, de pruebas que se relacionaran con las matemáticas abordadas en los seis módulos podría haber producido resultados distintos. Reconocemos que esta discusión es necesariamente especulativa, y tal vez se le considere como una "excusa" por no haber habido un impacto significativo. No obstante, a partir de nuestras encuestas a docentes, parece muy claro que la implementación de SM se vio obstaculizada, especialmente en su segundo año, por dos factores fuera del control de la innovación y algo independientes de la misma: las importantes pruebas nacionales en matemáticas, y la enseñanza de SM por parte de maestros con poco o ningún desarrollo profesional. En relación con el primer punto, los datos de la encuesta mostraron que la fidelidad de la implementación en el segundo año se redujo drásticamente, como lo demuestra cuánto se abarcó y dedicó tiempo al currículo, y la participación limitada en desarrollo profesional. Las observaciones hechas en visitas a las escuelas dejaron claro que el tiempo dedicado a SM se vio afectado negativamente por la atención prestada a las pruebas nacionales en matemáticas del final del año escolar, con más y más tiempo dedicado a la revisión y la práctica. De hecho, el equipo de SM descubrió, a partir de los datos de la encuesta, que al menos 25 escuelas habían dejado de enseñar SM tan temprano como a principios de enero del segundo año para dar espacio a la revisión de las matemáticas, en lugar de continuar hasta que se realizaran las pruebas Key Stage 2 en mayo. Además, debido a que el desarrollo profesional se midió a nivel escolar, es posible que en una escuela de alta fidelidad donde se cambió a un maestro que no había enseñado SM en el quinto grado, represente un caso en el que un inconveniente se convirtió en una mutación letal. En el peor de los casos, es posible que algunos maestros que trabajaron el currículo SM de sexto 
grado, ino hayan participado en ninguna capacitación de SM, ni hayan recibido ningún tipo de apoyo profesional en la escuela!

\section{OBSERVACIONES FINALES}

El proyecto SM fue ambicioso en sus objetivos, y quizás demasiado ambicioso en el rango de temas que se re-trabajaron en la estructura del micromundo. Pero, por mucho, el hallazgo más significativo fue la extrema susceptibilidad de la intervención a las condiciones iniciales de la experiencia, las competencias y la continuidad del docente. Creemos que este resultado se generaliza en entornos de aprendizaje basados en la idea del micromundo.

El problema es que a medida que aumentan las oportunidades de aprendizaje colaborativo, la interacción fluida y flexible, y el acceso a la información, no hay garantía de que éstos mejoren el aprendizaje: de hecho, la perspectiva de que los niños trabajen completamente solos sin maestros puede resultar económicamente atractiva aunque desde un punto de vista educativo, no lo es. A pesar de lo mucho que preferiríamos fuera de otro modo, el estudio de SM señala el hecho innegable de que la pedagogía y el currículo son elementos indispensables y esenciales de los micromundos -el fracaso en este sentido, sea por el motivo que fuera, invita a que cualquier intervención se transforme en una mutación letal.

Esto sólo se ve agravado por el impulso de cambio tecnológico. A medida que aumentan las oportunidades de aprendizaje colaborativo, de interacción fluida y flexible, y de acceso a la información, no hay garantía de que éstos mejoren el aprendizaje. El enfoque aquí está en la creación de artefactos culturalmente interesantes y resonantes, que simultáneamente brinden a los alumnos la oportunidad de toparse con ideas computacionales poderosas.

Hemos emergido de una era de teoría y práctica educativa en la que el papel del maestro en el diseño de micromundos era tema de debate: ahora es más esencial que nunca. Los mecanismos que sustentan todo tipo de artefactos son cada vez menos visibles (no hay piezas reparables por el usuario), y es inconcebible que los estudiantes o sus maestros hagan justicia al potencial de la tecnología para el aprendizaje matemático sin un cambio en la cultura educativa más amplia, que reconozca la centralidad del docente y de sus contextos en la búsqueda de cambio. 


\section{REFERENCIAS}

Abelson, H. \& diSessa, A. (1981/1986). Turtle Geometry: The computer as a medium for exploring mathematics. [Geometría de la tortuga. El ordenador como medio de expresión de las matemáticas]. Cambridge, MA, EUA: MIT Press.

Bamberger, J. \& Hernandez, A. (1999). Impromptu: An interactive software application. [Impromptu: Un software interactivo]. Nueva York, NY, EUA: Oxford University Press.

Benton, L., Hoyles, C., Kalas, I. \& Noss, R. (2017). Bridging primary programming and mathematics: Some findings of design research in England. Digital Experiences in Mathematics Education, 3(2), 1-24. doi 10.1007/s40751-017-0028-x

Brown, A. L. \& Campione, J. C. (1996). Psychological theory and the design of innovative learning environments: On procedures, principles, and systems. Mawah, NJ, EUA: Lawrence Erlbaum Associates, Inc.

Clayson, J. E. (2008). Radical bricolage: building coherence in the liberal arts using art, modeling and language. International Journal of Education through Art, 4(2), 141-161. doi 10.1386/eta.4.2.141_1

Confrey, J., Hoyles, C., Jones, D., Kahn, K., Maloney, A.P., Nguyen, K.H., Noss, R., Pratt, D. (2009) Designing software for mathematical engagement through modeling. En C. Hoyles \& J.B. Lagrange (eds) Mathematics education and technology: Rethinking the terrain. New ICMI Study Series, Vol. 13 (pp. 19-45). Boston, MA, EUA: Springer. doi 10.1007/978-1-4419-0146-0_3

diSessa, A. (2006). A history of conceptual change research: Threads and fault lines. En R. K. Sawyer (Ed.), The Cambridge Handbook of the Learning Sciences (pp. 265-281). Cambridge, Reino Unido: Cambridge University Press.

Edwards, L. D. (1998). Embodying mathematics and science: Microworlds as representations. Journal of Mathematical Behaviour, 17(1), 53-78.

Eisenberg, M. (2003). Mindstuff: educational technology beyond the computer Convergence, 9(2), 29-53. doi 10.1177/135485650300900205

Gargarian, G. (1993). The Art of Design: Expressive Intelligence in Music. Ph.D. Thesis, Massachusetts Institute of Technology (MIT), Dept. of Architecture and Planning. Retrieved from http://hdl.handle.net/1721.1/12559

Harel, I. \& Papert, S. (Eds.). (1991). Constructionism: Research Reports and Essays 19851990 by the Epistomology and Learning Research Group, MIT. [Construccionismo: reportes de investigación y ensayos del grupo de investigación de epistemología y aprendizaje del MIT. Norwood, New Jersey, EUA: Ablex Publishing Corporation. 
Harvey, B. (1997). Computer science Logo style volume 1: Symbolic computing. [Programación computacional estilo Logo volúmen 1: computación simbólica]. Cambridge, MA, EUA: MIT Press.

Hoyles, C. (1993). Microworlds/schoolworlds: The transformation of an innovation Learning from Computers: Mathematics Education and Technology (NATO ASI Series F, vol. 121) (pp. 1-17). Berlín, Alemania: Springer-Verlag.

Hoyles, C., Noss, R. \& Kent, P. (2004). On the integration of digital technologies into mathematics classrooms. International Journal of Computers for Mathematical Learning, 9(3), 309-326. doi 10.1007/s10758-004-3469-4

Monaghan, J., Trouche, L. \& Borwein, J. (2016). Tools and mathematics: Instruments for learning. [Herramientas y matemáticas: Instruments para el aprendizaje]. Nueva York, EUA: Springer. doi 10.1007/978-3-319-02396-0

Noss, R. \& Hoyles, C. (1996). Windows on mathematical meanings: Learning cultures and computers. [Ventanas a significados matemáticos: Aprendizaje de culturas y computadoras]. Dordrecht, Países Bajos: Kluwer Academic.

Noss, R. \& Hoyles, C. (2006). Exploring mathematics through construction and collaboration. En R. K. Sawyer (Ed.), The Cambridge Handbook of the Learning Sciences (pp. 389-405). Cambridge, Reino Unido: Cambridge University Press.

Papert, S. (1980/1981). Mindstorms: Children, computers, and powerful Ideas. [Desafío a la mente: computadoras y educación]. Nueva York: Basic Books.

Papert, S. (1991/2002). Situating constructionism. [Situar el construccionismo (Trad. INCAE)]. En I. Harel \& S. Papert (Eds.), Constructionism: Research reports and essays 1985-1990 by the epistemology and learning research group, MIT. Norwood, N.J.: Ablex Pub. Corp.

Papert, S. (1993/1995). The Children's Machine: Rethinking School In The Age Of The Computer [La máquina de los niños. Replantearse la educación en la era de los ordenadores]. Nueva York: Basic Books.

Papert, S. (2006). After how comes what. En R. K. Sawyer (Ed.), The Cambridge Handbook of the Learning Sciences. (pp. 581-586.). Cambridge, Reino Unido: Cambridge University Press.

Resnick, M. (1995). New paradigms for computing, new paradigms for thinking. En A. diSessa, C. Hoyles \& R. Noss (Eds.), Computers and exploratory learning. NATO ASI Series F, Volume 146 (pp. 31-43). Berlín, Alemania: Springer-Verlag.

Sacristán, A. I. \& Noss, R. (2008). Computational construction as a means to coordinate representations of infinity. International Journal of Computers for Mathematical Learning, 13(1), 47-70. doi 10.1007/s10758-008-9127-5 
Sinclair, N. \& Crespo, S. (2006). Learning mathematics in dynamic computer environments. Teaching Children Mathematics, 12(9), 436-444.

von Glasersfeld, E. (1989). Constructivism in education. En T. Husen \& P. T. N. (Eds.), International Encyclopedia of Education (Vol. 1, pp. 161-162). Oxford/Nueva York: Pergamon Press.

Wilensky, U. \& Papert, S. (2010). Restructurations: Reformulations of knowledge disciplines through new representational forms. En J. Clayson \& I. Kalas (Eds.), Constructionist approaches to creative learning, thinking and education: Lessons for the 21st century. Proceedings for Constructionism 2010 / The 12th EuroLogo conference (pp. 1-15). Paris, France: AUP. Retrieved from http://ccl.northwestern.edu/2010/wilensky_restructurations_Constructionism\%202010-latest.pdf

RICHARD NOSS

Domicilio: UCL Knowledge Lab

UCL Institute of Education, University College London

23- 29 Emerald Street

London WC1N 3QS, UK 